\title{
Systematic Review of Neuropsychological Outcomes in Dementia from Cognition- Based Psychological Interventions
}

\author{
Aimee Spector Martin Orrell Louise Hall \\ Research Department of Clinical Educational and Health Psychology, University College London, London, UK
}

\author{
Key Words \\ Cognition · Dementia • Neuropsychology • Cognitive \\ training $\cdot$ Cognitive stimulation
}

\begin{abstract}
Background/Aims: Although there is increasing evidence for the effectiveness of cognition-based psychological interventions in dementia, little is known about which neuropsychological domains are more amenable to change. Method: A systematic search identified randomised controlled trials grouped according to intervention type (cognitive training/ cognitive stimulation). Methodological quality was evaluated. Results: Of the 129 studies identified, 18 met the inclusion criteria; 11 were 'Cognitive Training' and 7 'Cognitive Stimulation'. For Cognitive Training, it was not possible to conclude which (if any) domains are most amenable to change. For Cognitive Stimulation, there was good evidence for general cognitive enhancement, more specifically in language and memory. Conclusions: Further in-depth trials are needed to determine neuropsychological processes more clearly.
\end{abstract}

Copyright $\odot 2012$ S. Karger AG, Basel

\section{Introduction}

In recent years, psychosocial interventions for dementia have become widely used and evaluated [1]. The development of psychological cognitive interventions for de- mentia was originally stimulated by evidence from the neuroscience literature. This suggested that the adult brain retains significant neuronal plasticity and therefore has the capacity for regeneration and compensation [25]. 'Dementia' encompasses a variety of disorders involving impaired cognition including memory, language, praxis, visuospatial and perceptual function and executive functions such as planning, organising and sequencing. Three main categories of cognition-focused psychological interventions have been described [6]: (1) cognitive training involves guided practice on a set of standard tasks designed to promote particular cognitive functions such as memory, attention or executive function, with the assumption that regular practice may improve or maintain functioning in that domain, (2) cognitive rehabilitation describes interventions that aim to build on preserved areas of cognition by developing strategies or using external aids to compensate for functional difficulties and (3) cognitive stimulation employs a range of activities and discussions aimed at the general enhancement of cognitive functioning, where tasks are more general, emphasise implicit as opposed to explicit learning and usually depend on the integration of various cognitive functions (e.g. memory, attention, language and problemsolving). Cognitive training and cognitive rehabilitation tend to be individualised approaches that are guided by needs, for example, it may be important for an individual to be able to dress themselves or learn the names of friends at a day centre. In contrast, cognitive stimulation usually

\section{KARGER \\ Fax +4161306 1234 \\ E-Mail karger@karger.ch}

www.karger.com (c) 2012 S. Karger AG, Basel

$1420-8008 / 12 / 0344-0244 \$ 38.00 / 0$

Accessible online at:

www.karger.com/dem
Dr. Aimee Spector

Research Department of Clinical

Educational \& Health Psychology, University College London

1-19 Torrington Place, London WC1E 7HB (UK)

E-Mail a.spector@ucl.ac.uk 
runs in a group format, often following a programme known as 'CST' [cognitive stimulation therapy, see [40]).

Existing systematic reviews evaluating the efficacy of psychological cognitive interventions in dementia have generally only looked at change on the most widely used, generic measures of cognition, i.e. the Mini Mental State Examination (MMSE) and the cognitive subscale of the Alzheimer's Disease Assessment Scale (ADAS-Cog) [79]. One review looked at change on more specific measures of cognitive function [7], but unfortunately grouped cognitive training and cognitive stimulation with other psychological interventions, labelling them all as 'restorative strategies' and not exploring differential effects on cognition. Our review aims to identify which neuropsychological domains show improvements or maintenance of function in people with mild to moderate dementia in relation to participation in cognition-focused psychological interventions.

\section{Methods}

\section{Inclusion Criteria}

Only randomised controlled trials (RCTs) published in English in peer-reviewed journals were considered for inclusion. Additional criteria relating to study participants, interventions and outcome measures were used to select studies which met the quality criteria and were relevant to the aim.

\section{Type of Participants}

- Diagnosed with dementia using validated, systematic diagnostic criteria such as DSM-IV-TR [10] or ICD-10 [11].

- Mild to moderate dementia as estimated by a standardised measure such as the MMSE [12] or Clinical Dementia Rating (CDR) [13].

\section{Types of Interventions}

- Aimed primarily at improving or maintaining cognitive function: techniques, therapy, groups, training, strategies or stimulation that directly and explicitly target cognitive functioning.

- Acceptable control conditions including 'no treatment', 'treatment as usual', another active treatment or placebo.

\section{Types of Outcome Measures}

- Evaluated in terms of change from baseline on at least one standardised measure of cognitive function.

Search Methods for Identification of Studies

Studies were identified by searching ALOIS (www. medicine. ox.ac.uk/alois), the specialized register of the Cochrane Dementia and Cognitive Improvement Group including records from a range of sources i.e. Medline, Embase, PsycINFO and Cinahl. No start date was used for the search in order to include as many studies as possible. The search was limited to RCTs. Search terms were 'dementia' or 'Alzheimer disease' and at least one of the following: 'cognitive', 'memory', 'reality', 'orientation', 'stimulation', 'rehabilitation', 'training', 'remediation' and 'retraining'. Additionally, the terms were entered into PsycINFO. Abstracts were read to determine whether each study met inclusion criteria.

\section{Categorisation of Studies}

The above definitions of 'Cognitive Stimulation' and 'Cognitive Training' were used [6]. None of the studies eligible for inclusion involved 'Cognitive Rehabilitation', hence it was excluded. Scores on most neuropsychological tests can be affected by multiple cognitive abilities; however, a primary ability is usually identifiable. Measures were grouped by the primary domain of cognition evaluated. Measures for which the primary domain could not be identified due to lack of information (e.g. Syndrom Kurztest) were excluded. The methodological quality of each study was objectively rated using the Jadad scale for evaluating RCTs [14], which gives studies up to 5 points according to randomisation, blinding, whether dropouts are described, method of randomisation and method of double-blinding. A study was designated 'high quality' if it scored 3-5, 'medium quality' if it scored 2 and 'low quality' if it scored 0 or 1 .

\section{Results}

Searches identified 129 potential studies, 18 of which met the inclusion criteria, 11 being cognitive training and 7 being cognitive stimulation. A heterogeneous range of measures was used to evaluate cognitive change. The two most frequently used measures were the MMSE [12] and the ADAS-Cog [15]. Both are used as measures of global cognitive ability. The MMSE is a brief measure used clinically to screen for cognitive impairment. It contains items that assess orientation to time and place, attention and calculation, language and immediate and delayed recall. The ADAS-Cog is frequently used as a primary efficacy measure in clinical trials of medication for Alzheimer's disease [16]. It consists of three subscales: memory, praxis and language; however, only the overall score out of 70 tends to be reported.

\section{Cognitive Training Interventions}

Full details of the 11 cognitive training interventions can be found in tables 1 and 2. Four were rated as highquality, 3 as medium-quality and 4 as low-quality. Studies varied somewhat in content, both for the treatment and comparison groups. In terms of comparison groups, 2 studies used a waiting-list control, 2 used a treatment-asusual control group and 1 used two comparison groups: medication only and placebo medication. The remaining 6 studies all used a placebo comparison group in order to control for general effects of therapist attention and/or social interaction. Five studies evaluated cognitive train- 


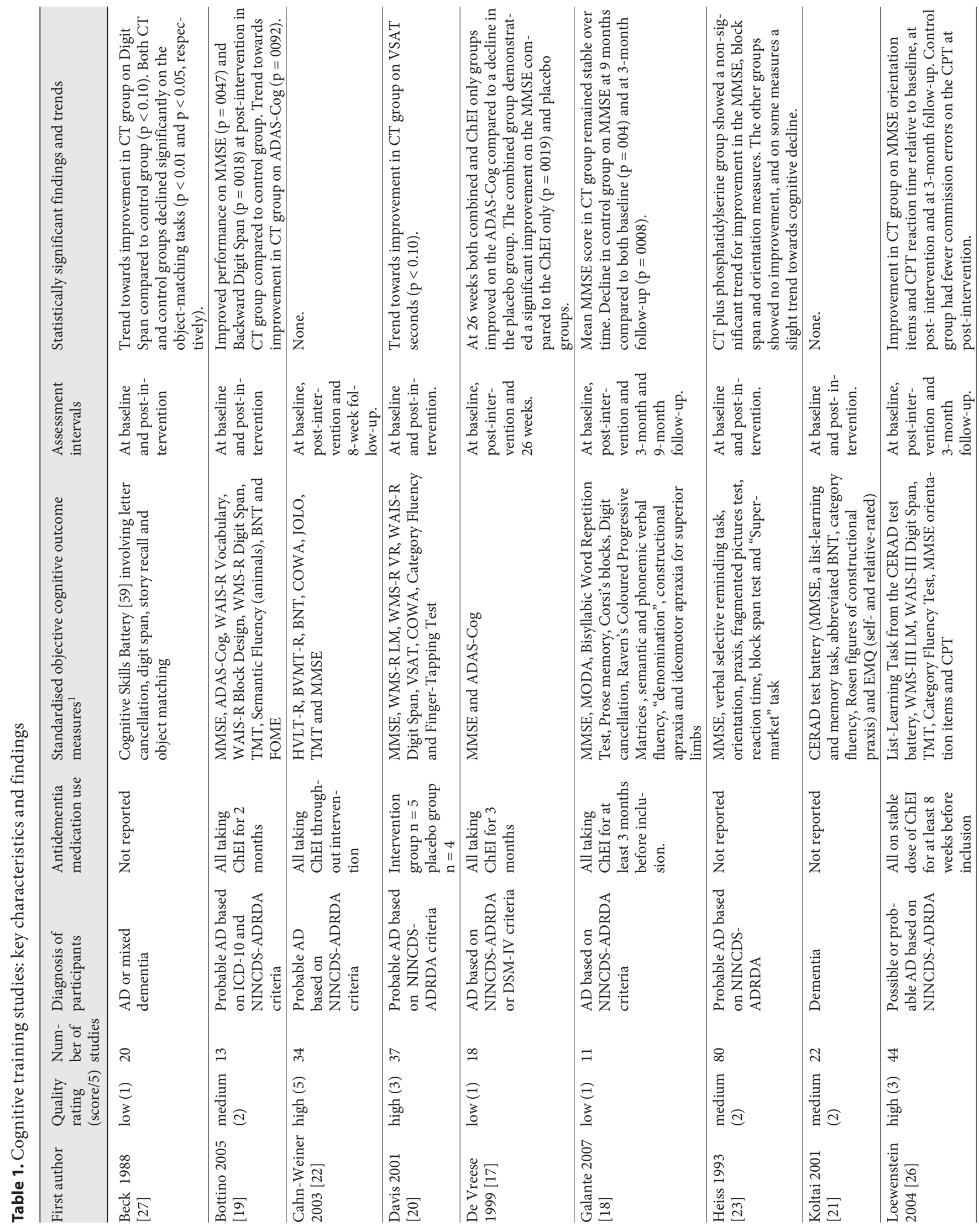




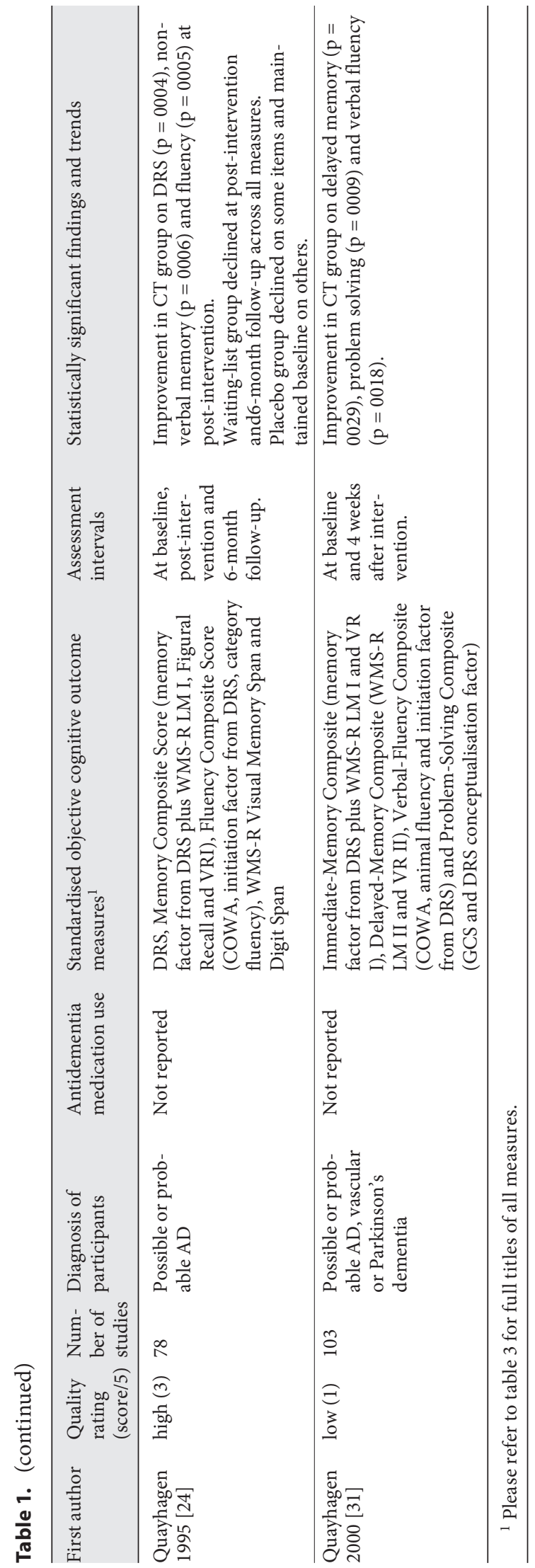

Cognition-Based Interventions for Dementia: Systematic Review of Neuropsychological Outcomes ing in combination with acetylcholinesterase inhibitors (ChEIs). Studies also varied significantly in terms of length of intervention (range 4-24 weeks, $\mathrm{M}=10.5, \mathrm{SD}=$ 6.6), number of sessions delivered (range $5-72, M=25.3$, $\mathrm{SD}=20.9$ ) and mode of delivery. Some interventions were delivered by a clinician or member of the research team, some by computer and some using a combination of professional and family-caregiver facilitation. Three studies looked at group interventions, whereas the remaining studies looked at individual interventions.

General Cognitive Function. Eight of the 11 studies used a measure of general cognitive function as an outcome measure, with 7 using the MMSE and 2 using the ADAS-Cog. Three studies provided some evidence for enhancement of general cognitive function, but 2 of these were rated as being of low methodological quality $[17,18]$ and 1 as being of medium quality [19]; these 3 were also the smallest out of all of the studies and 2 of them failed to control for general effects such as therapist attention and social interaction $[17,19]$. There were no significant differences in the remaining 4 studies that employed the MMSE [20-23], 2 high-quality and 2 medium-quality studies. A 'high quality' study [24] used the Dementia Rating Scale [25] to measure general cognitive ability, finding statistically significant improvements after intervention relative to both waiting-list and placebo-control groups. However, baseline data for each of the groups were not reported and so the findings could also be due to group differences in baseline scores.

Learning and Memory. All but 1 [17] of the 11 studies of cognitive training interventions used at least one test of learning and memory as an outcome measure. The aforementioned high-quality study [24] found significant improvements in non-verbal and delayed memory. One study [20] found that both intervention and control groups (consisting of unstructured conversation, questioning and reciting overlearned material) improved significantly on verbal and non-verbal memory. They suggested that these findings may be due to small practice effects. In the remaining studies, no significant changes in measures of learning and memory were found; although 2 of these were rated as being of high quality [22, 26], they all had relatively small sample sizes and may therefore have lacked power to detect an effect.

Orientation. Only 2 studies measured orientation. One [26] trained participants on the orientation items from the MMSE and used the same items as an outcome measure, finding significantly higher scores at both the post-intervention and 3-month follow-ups. Another [23] did not find any statistically significant improvements in 


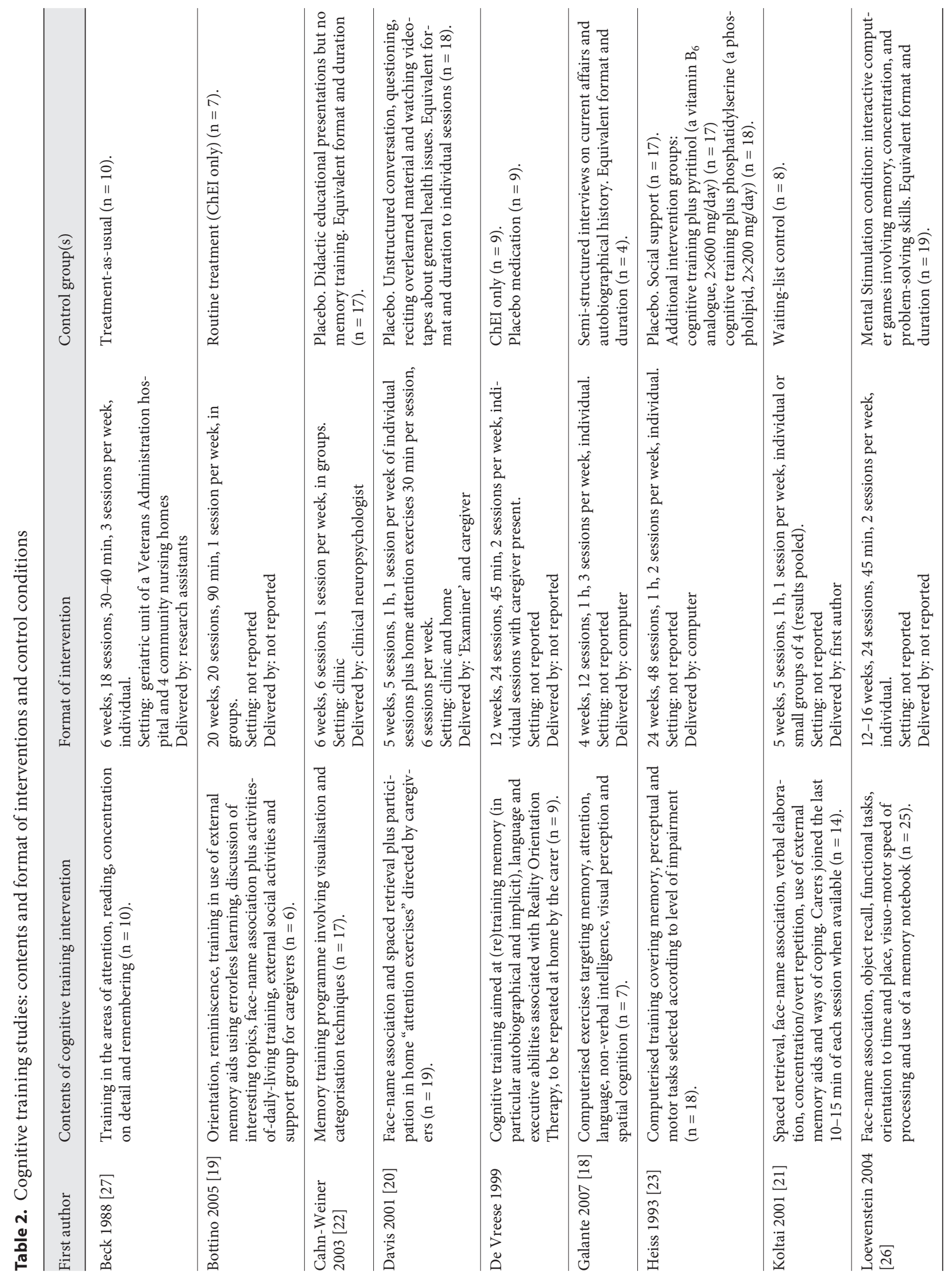




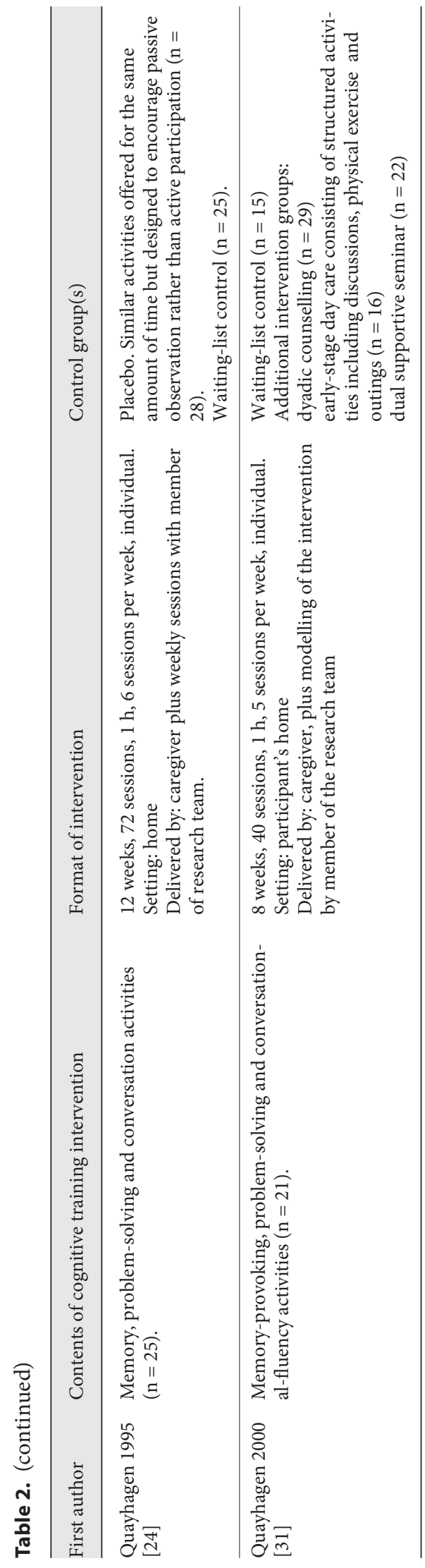

Cognition-Based Interventions for Dementia: Systematic Review of Neuropsychological Outcomes orientation following their cognitive training intervention.

Attention/Working Memory. Seven studies used a digit span test and/or a spatial span test as a measure of attention/working memory. Two $[19,27]$ found a significant improvement on a digit span task; however, one [27] was rated as low-quality and there were statistically significant differences in baseline characteristics between groups despite randomisation. In the remaining studies, no significant differences were found. One study [20] used the Verbal Series Attention Test [28] as an additional measure of attention/working memory, finding insignificant but positive trends. Another [26] used the Continuous Performance Test (CPT) [29] as a further measure of attention/working memory, with mixed results. Participants in the intervention group showed significant improvements in the time score of the CPT at both the post-intervention and 3-month follow-up. However, the number of commission errors appeared to increase significantly whereas the placebo group achieved a significantly lower number of commission errors at the postintervention follow-up. It is important to note that they [26] used the CPT as one of the training tasks in their cognitive intervention.

Language. Only 4 studies investigated the impact of cognitive training on language. Three $[19,21,22]$ found no significant differences between intervention and control groups on the Boston Naming Test [30]. One [18] did not find any significant changes on a word repetition task. Of note, these were all relatively small studies that may have lacked power to detect an effect.

Executive Function. Verbal fluency tasks were used as outcomes in 8 studies. Only 2 [24, 31] found a significant improvement in verbal fluency relative to the control group. Two [26, 19] used the Trail-Making Test [32], a measure of response inhibition and set-shifting, finding no significant differences. One [29] found a significant change over time on a problem-solving composite score for participants in their cognitive intervention group.

Praxis and Motor Function. Only 3 studies measured praxis as an outcome. One of the highest-quality studies [20] did not find any statistically significant group differences on the finger-tapping test which measures self-directed manual motor speed [33]. One study [21] did not report the outcomes on a measure of constructional praxis which they used as part of the CERAD (Consortium to Establish a Registry for Alzheimer's Disease test battery) [34], suggesting that results were not significant. Another [18] did not find any significant differences on measures of constructional and ideomotor apraxia; however, with 
a sample size of just 11, it is hard to draw conclusions from this study.

Visual Perception. A range of tests of visual perception were employed including Benton, Hannay and Varney's [35] Judgement of Line Orientation [22], a cancellation task [18], a fragmented-pictures test [23] and an objectmatching task [27]. None of these studies reported any significant findings for tests of visual perception.

\section{Cognitive Stimulation Interventions}

Full details of the 7 cognitive stimulation studies can be found in table 3. Studies varied somewhat in terms of the comparison group(s) used, with 1 using 'psychosocial support', 5 using 'treatment-as-usual' or 'no treatment' and 1 using basic stimulation and ChEI medication. Four studies evaluated cognitive stimulation in combination with a stable dose of ChEI [36-39]. Studies varied in terms of length of intervention (range 5-52 weeks; $M=19.9$, $\mathrm{SD}=16.2$ ) and number of sessions delivered (range 10$103 ; \mathrm{M}=51.7, \mathrm{SD}=33.9$ ). There was some heterogeneity in the mode of delivery, with 4 studies evaluating groups and 3 evaluating individual interventions. Most interventions were delivered by a clinician or member of the research team, but in 1 study the intervention was delivered by computer and in another by family caregivers.

General Cognitive Function. All 7 studies used at least one measure of general cognitive function as an outcome measure and all used the MMSE. Five of these found significant results in the intervention group compared to the controls. The ADAS-Cog was employed as an additional outcome measure in 4 studies, all finding significant improvements [36, 37, 39, 40]. One study [41] measured general cognitive function using the Kingston Dementia Rating Scale [42] and found that both their cognitivestimulation ('enhanced reality orientation') and 'social interaction' groups showed significant improvements 1 week after intervention, returning to baseline levels at 10 weeks. The treatment-as-usual control group's scores did not change over time. Six out of the 7 studies, including the highest-quality, most methodologically rigorous studies $[36,37,40]$ found positive effects on at least one measure of general cognitive functioning.

Learning and Memory. One study [3] found a non-significant trend towards improvement on both a word-list memory task and an 'association' test in their cognitive stimulation group. Another [43] analysed the memory subscale of the ADAS-Cog but found no statistically significant effects. This subscale is, however, based solely on a word-list learning and recall item which may lack ecological validity. The remaining studies of cognitive stim- ulation interventions did not use detailed measures of learning and memory.

Language. One trial [3] did not analyse the results of the naming section of the CERAD test battery [33] due to a high proportion of ceiling effects. For another trial [39], a subsequent analysis of the significant ADAS-Cog changes [43] showed that, of the three subscales, 'language' was the only one to show significant changes. Within this subscale, 'spoken language' and 'commands' changed significantly.

Executive Function. Only 1 trial was evaluated for this [3]; verbal fluency was measured and no significant group differences were reported.

Praxis. Only 1 trial [3] used a specific measure of praxis. The results could not be analysed due to a ceiling effect. Another study [40] found no significant effects on the praxis subscale of the ADAS-Cog.

\section{Discussion}

It is not possible to state with any certainty which cognitive domains show improvements or maintenance of function in people with dementia participating in cognition-focused psychological interventions. In cognitive training research, more attempts have been made to examine changes in objective cognitive measures, rather than solely relying on measures of general cognitive function. Learning and memory, attention/working memory and the initiation aspect of executive function are the most evaluated areas, yet results are inconclusive in all three areas. In terms of other dementia-relevant cognitive domains (praxis, orientation, visual perception, language and other aspects of executive function) more work is needed to determine whether improvement or maintenance of function is feasible. The studies to date have been significantly limited by small sample sizes, with reduced statistical power. It is difficult to ascertain whether the content of the intervention was linked to outcome, as there are so many confounding factors such as mode of delivery, length of intervention and study quality. There were only 2 high-quality studies with significant results [26, 24]; the former described their activities in detail (e.g. face-name association, object recall, functional tasks and orientation) and the latter more broadly (memory, problem-solving and conversation), yet it may be that there was some overlap between these two interventions.

Two large, methodologically rigorous RCTs of cognitive stimulation have been conducted $[37,40]$, both find- 


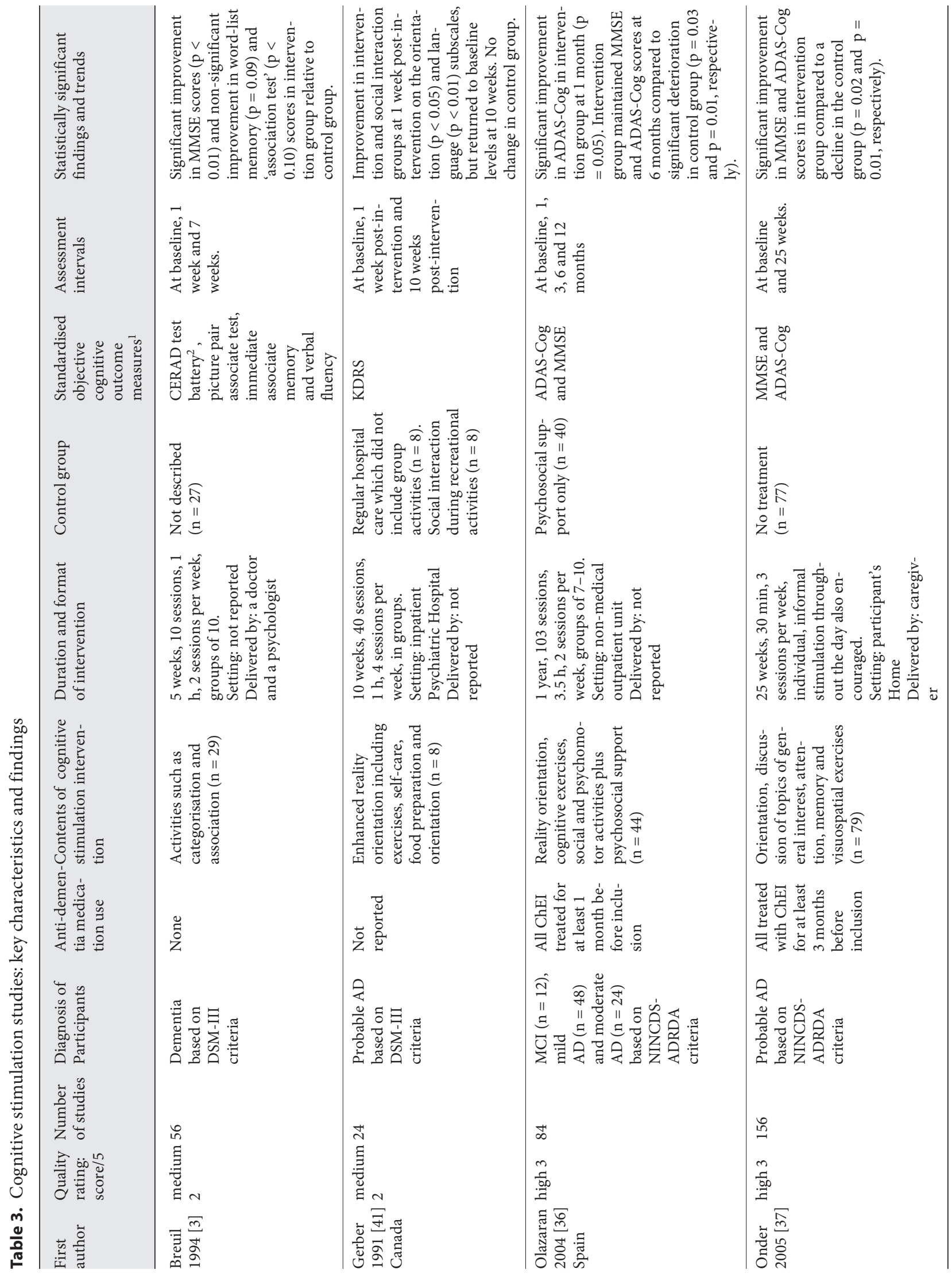




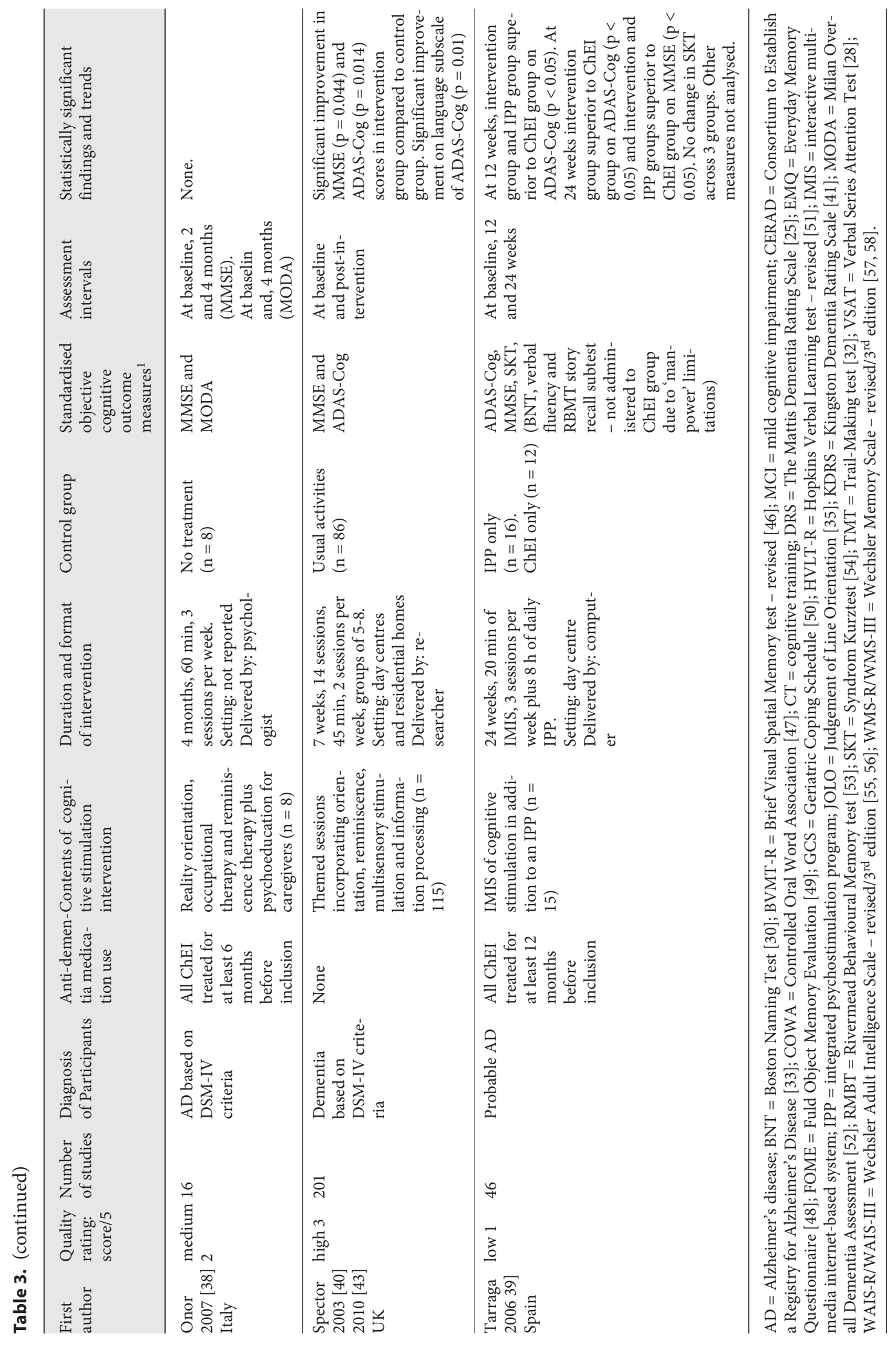


ing significant improvements in general cognitive function. This finding has been supported by the smaller studies. There is robust evidence for improved cognitive functioning for people with dementia using cognitive stimulation, confirmed by the recent Cochrane review [9]. Only 1 study provided results for more detailed neuropsychological outcome measures [3] and another attempted to examine the subscales of the ADAS-Cog [43]. Results suggest that language and possibly memory may be the cognitive domains most amenable to change following cognitive stimulation.

\section{Limitations}

Neuropsychological tests are not necessarily indicative of the effects that these interventions may have on cognitive performance in everyday life, and the ecological validity of neuropsychological tests is typically moderate [44]. Moreover, it is not always evident how a change in a measure will translate practically for an individual. In addition, assumptions about the quality of the studies were based solely on the information provided and methodological details were often omitted from the report.

\section{Further Research}

Larger scale, trials of quality are needed to further investigate the neuropsychological outcomes of cognitive training and cognitive stimulation, in order to increase the understanding of which aspects of cognition are more amenable to change. It has been suggested [7] that the aim of cognitive rehabilitation is to improve function and/or wellbeing, rather than to improve performance on cognitive tests per se. This may imply that functional outcomes reflecting individualised rehabilitation goals may be more suitable than further neuropsychological investigations. Future trials should use more sensitive neuropsychological tests. Participation in stimulating activities may lead to enhanced activation of neurons in the brain. Therefore, improved performance in cognitive function could reflect enhanced functioning of the existing neuronal networks typically used to perform these tasks or enhanced recruitment of alternative neuronal networks [5]. Functional brain imaging studies are required to further explore these hypotheses. Another recommended area for future research is the development of a more suitable and comprehensive neuropsychological test battery for people with mild to moderate dementia. There are weaknesses in existing batteries; the CERAD [34], for example, may be prone to floor and ceiling effects [3].

\section{Conclusion}

This review does not specifically indicate which aspects of cognitive performance are most likely to improve following cognitive stimulation or cognitive training. Nonetheless, there is strong evidence to support the widespread clinical use of cognitive stimulation, and this is advocated in UK government guidelines [45]. Research investigating the optimum implementation of cognitive stimulation would be beneficial, as the content, frequency, duration, format, delivery mode and number of sessions of the interventions in this review varied considerably. It would be helpful to know which strategies and activities are the most important, in order to implement these in clinical practice.

\section{References}

1 Olazaran J, Reisberg B, Cruz I, Pena-Cassanova J, Teodoro S, Woods B, Beck C, Auer S, Lai C, Spector A, Fazio S, Mittleman M, Bond J, Kivipelto M, Brodaty H, Teri L, Rojo JM, Orrell M, Feldman H, Muniz R: Nonpharmacological therapies in Alzheimer's disease: a systematic review of efficacy. Dement Geriatr Cogn Disord 2010;30:161-178.

-2 Becker JT, Mintum MA, Aleva K, Wiseman MB, Nichols T, DeKosky ST: Compensatory reallocation of brain resources supporting verbal episodic memory in Alzheimer's disease. Neurology 1996;46:692-700.

3 Breuil V, De Rotrou J, Forette F, Tortrar D, Ganancia-Ganem A, Frambourt A: Cognitive stimulation of patients with dementia: preliminary results. Int J Geriatr Psychiatry 1994;9:211-217.
4 Raisman G: Neuronal plasticity in the septa1 nuclei of the adult brain. Brain Res 1969; 14:25-48.

5 Stern Y, Moeller JR, Anderson KE, Luber B, Zubin NR, DiMauro AA, et al: Different brain networks mediate task performance in normal aging and $\mathrm{AD}$ : defining compensation. Neurology 2000;55:1291-1297.

6 Clare L, Woods RT: Cognitive training and cognitive rehabilitation for people with early-stage Alzheimer's disease: a review. Neuropsychol Rehabil 2004;14:385-401.
7 Clare L, Woods RT, Moniz Cook ED, Orrell $\mathrm{M}$, Spector A: Cognitive rehabilitation and cognitive training for early-stage Alzheimer's disease and vascular dementia. Cochrane Database Syst Rev 2003;4:CD003260.

-8 Sitzer DI, Twamley EW, Jeste DV: Cognitive training in Alzheimer's disease: a meta-analysis of the literature. Acta Psychiatrica Scand 2006;114:75-90.

9 Woods B, Aguirre E, Spector A, Orrell M: Can cognitive stimulation benefit people with dementia (review). Cochrane Database Syst Rev 2012, E-pub ahead of print.

10 American Psychiatric Association: Diagnostic and Statistical Manual of Mental Disorders, ed 4. Washington, American Psychiatric Association, 2000. 
11 World Health Organisation: The ICD-10 Classification of Mental and Behavioural Disorders: Clinical Descriptions and Diagnostic Guidelines 1992. Geneva, World Health Organisation, Division of Mental Health.

-12 Folstein MF, Folstein SE, McHugh PR: Minimental state: a practical method for grading the cognitive state of patients for the clinician. J Psychiatric Res 1975;12:189-198.

-13 Hughes C, Berg L, Danziger W, Coben L, Martin R: A new clinical scale for the staging of dementia. Br J Psychiatry 1982;140:566-572.

14 Jadad AR, Moore RA, Carroll D, Jenkinson C, Reynolds DJ, Gavaghan DJ, et al: Assessing the quality of reports of randomized clinical trials: is blinding necessary? Control Clin Trials 1996;17:1-12.

15 Rosen WG, Mohs RC, Davis KL: A new rating scale for Alzheimer's disease. Am J Psychiatry 1984;141:1356-1364.

- 16 Rockwood K, Fay S, Gorman M, Carver D, Graham JE: The clinical meaningfulness of ADAS-Cog changes in Alzheimer's disease patients treated with donepezil in an openlabel trial. BMC Neurol 2007;7:26-33.

17 De Vreese LP, Neri M: Ecological impact of combined cognitive training programs (CTP) and drug treatment (ChE-I) in AD. Int Psychogeriatr 1999;11(suppl):S187.

18 Galante E, Venturini G, Fiaccadori C: Computer-based cognitive intervention for dementia: preliminary results of a randomized clinical trial. G Ital Med Lav Ergon 2007;29:B26-B32.

-19 Bottino CMC, Carvalho IAM, Alvarez AM, Avila R, Zukauskas PR, Bustamante S, et al: Cognitive rehabilitation combined with drug treatment in Alzheimer's disease patients: a pilot study. Clin Rehabil 2005;19:861-869.

20 Davis RN, Massman PJ, Doody RS: Cognitive intervention in Alzheimer disease: a randomized placebo-controlled study. Alzheimer Dis Assoc Disord 2001;15:1-9.

-21 Koltai DC, Welsh-Bohmer KA, Schmechel DE: Influence of anosognosia on treatment outcome among dementia patients. Neuropsychol Rehabil 2001;11:455-475.

-22 Cahn-Weiner DA, Malloy PF, Rebok GW, Ott BR: Results of a randomized placebocontrolled study of memory training for mildly impaired Alzheimer's disease patients. Appl Neuropsychol 2003;10:215-223.

-23 Heiss WD, Kessler J, Slansky I, Mielke R, Szelies B, Herholz K: Activation PET as an instrument to determine therapeutic efficacy in Alzheimer's disease. Ann NY Acad Sci 1993;695:327-331.

24 Quayhagen MP, Quayhagen M, Corbeil RR, Roth PA, Rodgers JA: A dyadic remediation program for care recipients with dementia. Nursing Res 1995;44:153-159.
Coblentz JM, Mattis S, Zingesser H, Kasoff SS, Wisniewski HM, Katzman R: Presenile dementia: clinical aspects and evaluation of cerebro-spinal fluid dynamincs. Arch Neurol 1973;29:299-308.

26 Loewenstein DA, Acevedo A, Czaja SJ, Duara R: Cognitive rehabilitation of mildly impaired Alzheimer disease patients on cholinesterase inhibitors. Am J Geriatr Psychiatry 2004;12:395-402.

27 Beck C, Heacock P, Mercer S, Thatcher R, Sparkman C: The impact of cognitive skills remediation training on persons with $\mathrm{Al}$ zheimer's disease or mixed dementia. J Geriatr Psychiatry 1988;21:73-88.

28 Mahurin RK, Cooke N: The Verbal Series Attention Test: normal and demented older adults. Clin Neuropsychol 1996;10:43-53.

29 Conners CK: Conner's Continuous Performance Test. II. Odessa, Psychological Assessment Resources, 2007.

30 Kaplan EF, Goodglass H, Weintraub S: The Boston Naming Test, ed 2. Philadelphia, Lippincott, Williams \& Wilkins, 2001.

- 31 Quayhagen MP, Quayhagen M, Corbeil RR, Hendrix RC, Jackson JE, Snyder L, et al: Coping with dementia: evaluation of four nonpharmacologic interventions. Int Psychogeriatr 2000;12:249-265.

32 Reitan RM, Wolfson D: The Halstead-Reitan Neuropsychological Test Battery: Theory and Clinical Interpretation, ed 2. Tucson, Neuropsychology Press, 1992.

33 Strauss E, Sherman EMS, Spreen O: A Compendium of Neuropsychological Tests, ed 3. Oxford, Oxford University Press, 2006.

34 Morris JC, Heyman A, Mohs RC, Hughes J, Van Belle G, Fillenbaum, et al: The consortium to establish a registry for Alzheimer's disease (CERAD). Part I. Clinical and neuropsychological assessment of Alzheimer's disease. Neurology 1989;39:1159-1165.

35 Benton A, Hannay HJ, Varney NR: Visual perception of line direction in patients with unilateral brain disease. Neurology 1975;25: 907-910.

36 Olazarán J, Muñiz R, Reisberg B, Peña-Casanova J, del Ser T, Cruz-Jentoft AJ, et al: benefits of cognitive-motor intervention in MCI and mild to moderate Alzheimer disease. Neurology 2004;63:2348-2353.

37 Onder G, Zanetti O, Giacobini E, Frisoni GB, Bartorelli L, Carbone G, et al: Reality orientation therapy combined with cholinesterase inhibitors in Alzheimer's disease: randomised controlled trial. Br J Psychiatry 2005; 187:450-456.
38 Onor ML, Trevisiol M, Negro C, Alessandra S, Saina M, Aguglia E: Impact of a multimodal rehabilitative intervention on demented patients and their caregivers. Am J Alzheimers Dis Other Demen 2007;22:261-272.

-39 Tarraga L, Boada M, Modinos G, Espinosa A, Diego S, Morera A, et al: A randomised pilot study to assess the efficacy of an interactive, multimedia tool of cognitive stimulation in Alzheimer's disease. J Neurol Neurosurg Psychiatry 2006;77:1116-1121.

40 Spector A, Thorgrimsen L, Woods B, Royan L, Davies S, Butterworth M: Efficacy of an evidence-based cognitive stimulation therapy programme for people with dementia: randomised controlled trial. Br J Psychiatry 2003;183:248-254.

41 Gerber G, Prince P, Snider HG, Atchison K, Dubois L, Kilgour JA: Group activity and cognitive improvement among patients with Alzheimer's disease. Hosp Community Psychiatry 1991;42:843-845.

42 Pellietier F, Hopkins R, Hamilton P: The Kingston Dementia Rating Scale. Int J Geriatr Psychiatry 1991;6:227-233.

43 Spector A, Orrell M, Woods B: Cognitive Stimulation Therapy (CST): effects on different areas of cognitive function for people with dementia. Int J Geriatr Psychiatry 2010; 25:1253-1258.

44 Chaytor N, Schmitter-Edgecombe M: The ecological validity of neuropsychological tests: a review of the literature on everyday cognitive skills. Neuropsychol Rev 2003;13: 181-197.

45 NICE-SCIE: Dementia: supporting people with dementia and their carers in health and social care. NICE Clinical Guideline 42. London, National Institute for Health and Clinical Excellence, 2007. www.nice.org.uk.

-46 Benedict RHB, Schretlen D, Groninger L, Dobraski M, Shpritz M: Revision of the Brief Visuospatial Memory Test: studies of normal performance, reliability, and validity. Psychol Assess 1996;8:145-153.

47 Benton AL: Differential behavioural effects in frontal lobe disease. Neuropsychologia 1968;6:53-60.

48 Sunderland A, Harris JE, Baddeley AD: Do laboratory tests predict everyday memory? A neuropsychological study. J Verbal Learn Verbal Behav 1983;22:727-738.

49 Fuld PA: Guaranteed stimulus-processing in the evaluation of memory and learning. Cortex 1980;16:255-272.

50 Quayhagen MP, Chiriboga D: Geriatric coping schedule; in Mangen DJ, Peterson WA (eds): Research Instruments in Social Gerontology: Clinical and Social Psychology, vol 1. Minneapolis, University of Minnesota Press, 1976, pp 168-169, pp 182-183. 
-51 Benedict RHB, Schretlen D, Groninger L, Brandt J: Hopkins Verbal Learning Test - revised: normative data and analysis of interform and test-retest reliability. Clin Neuropsychol 1998;12:43-55.

52 Brazzelli M, Capitani E, Della Sala S, Spinnler H, Zuffi M: A neuropsychological instrument adding to the description of patients with suspected cortical dementia: the Milan Overall Dementia Assessment. J Neurol Neurosurg Psychiatry 1994;57:1510-1517.
53 Wilson B, Cockburn J, Baddeley A: The Rivermead Behavioral Memory Test. Reading, Thames Valley Test Company, 1985.

54 Erzigkeit H: The SKT: a short cognitive performance test as an instrument for the assessment of clinical efficacy of cognitive enhancers; in Berenger M, Reisberg B (eds): Diagnosis and Treatment of Senile Dementia. Berlin, Springer Verlag, 1989, pp. 164-74.
55 Wechsler D: Wechsler Adult Intelligence Scale: revised edition. San Antonio, Psychological Corporation, 1981.

56 Wechsler D: Wechsler Adult Intelligence Scale: ed 3. San Antonio, Psychological Corporation, 1997.

57 Wechsler D: Wechsler Memory Scale: revised edition. San Antonio, Psychological Corporation, 1987

58 Wechsler D: Wechsler Memory Scale: ed 3. San Antonio, Psychological Corporation, 1997.

59 Carter LT, Caruso JL, Languirand MA, Berard MA: The Thinking Skills Workbook: a cognitive skills remediation manual for adults. Springfield, Charles C. Thomas, 1980. 